Open Access

\title{
Clinical and Economic Outcomes Associated with Low-Dose Fluticasone Propionate Versus Montelukast in Children with Asthma Aged 4 to 11 Years
}

\author{
Richard H. Stanford ${ }^{*}, 1$, Manan Shah ${ }^{2}$ and Sham L. Chaudhari ${ }^{2}$ \\ ${ }^{I}$ GlaxoSmithKline, Research Triangle Park, North Carolina, USA \\ ${ }^{2}$ Xcenda, Palm Harbor, Florida, USA
}

\begin{abstract}
Objective: Inhaled corticosteroids (ICS) are preferred first-line controller agents for adults and adolescents with asthma. There is limited effectiveness data comparing ICS to leukotriene receptor antagonists (LTRA) in children with asthma aged 4 to 11 years.
\end{abstract}

Methods: A retrospective, matched cohort study was conducted using medical and pharmacy claims data. Asthma patients (ICD-9, 493.xx) naïve to any asthma controller therapy, and having $\geq 1$ dispensing of fluticasone propionate $44 \mathrm{mcg}$ (FP44), an ICS, or montelukast any dose (MON), an LTRA, were identified. Drug cohorts were matched (1:2) using propensity scores. Outcomes during follow-up included asthma-related ED visits, composite measure of asthma-related ED/hospital visit, asthma-related costs per month, and monthly rescue medication use. Statistical differences between cohorts were evaluated using multivariate regression models.

Results: The final matched sample included 6,636 patients (FP44=2,212; MON=4,424). During follow-up, the FP44 cohort had a $29 \%$ significantly lower risk of an asthma-related ED visit (Hazard ratio $(95 \% \mathrm{CI})=0.71(0.52,0.96)$ ) compared to the MON cohort. Monthly asthma-related costs were significantly reduced on average by $36 \%$ in the FP44 compared to the MON cohort ( $\$ 48 v s \$ 75, \mathrm{p}<0.05)$. Use of short-acting beta-agonists per month were similar between cohorts but monthly adjusted number of oral corticosteroid prescriptions were significantly lower in the FP44 compared to the MON cohort (0.03 vs 0.04, $\mathrm{p}<0.001)$.

Conclusion: Initiation of FP44 versus MON in children with asthma aged 4 to 11 years is associated with a significant reduction in asthma-related ED visits, costs, and oral corticosteroid use.

Keywords: Asthma, pediatrics, inhaled corticosteroids, leukotriene, receptor antagonists, outcomes, costs.

\section{INTRODUCTION}

Asthma exerts a significant morbid toll on children as rates of asthma attacks leading to emergency department visits and hospitalizations are more than twice in children under 18 years of age compared to adults [1]. Furthermore, asthma is the second-ranking cause of all hospitalizations in children accounting for $7 \%$ of all stays for pediatric illnesses [2]. Therefore, the choice of appropriate, long-term controller therapy is paramount in this population. Inhaled corticosteroids (ICS) are currently recommended as the preferred controller medication for persistent asthma in children by national and international guidelines among other therapeutic options that includes leukotriene receptor antagonists (LTRAs), cromolyn, nedocromil, theophylline, and long-acting beta-agonists [3,4]. In comparison with no ICS treatment, treatment with ICS in children has been shown to improve pulmonary function, and decrease asthma exacerbations leading to hospitalizations and emergency department (ED) visits [5-7].

\footnotetext{
*Address correspondence to this author at the Five Moore Drive, Research Triangle Park, NC 27709, USA; Tel: 919483 3779; Fax: 919-315-5042; Email: richard.h.stanford@gsk.com
}

In head-to-head comparative, randomized controlled trials in children $>2$ years of age with other long-term controller therapy options such as LTRAs, ICSs have been shown to have superior efficacy [8-14]. Furthermore, ICS were observed to have better clinical efficacy when compared to LTRAs for the treatment of mild persistent asthma based on a Cochrane Review [15]. Observational studies provide the opportunity to assess the effect treatment on a population of patients that are prescribed treatments in clinical practice and several have observed a similar effect in real-world clinical [16-19]. In the most recent of these studies, fluticasone propionate (FP), an ICS was compared to an LTRA, montelukast (MON) in children with asthma aged 4-17 years who were previously receiving ICS therapy [17]. During the follow-up period, FP was found to be associated with better outcomes as the odds of experiencing treatment failure was more than two times for MON compared to the FP cohort (Odds ratio $[\mathrm{OR}]=2.55,95 \%$ confidence interval $[C I]=2.19$ to 2.96 ). Significant economic benefits in terms of reduced asthma-related costs were also found for FP compared to the MON cohort [19].

Stempel et al. evaluated a pediatric population between the ages of 4 to 17 years whereas recent National Asthma Education and Prevention Program (NAEPP) guidelines have redefined the ages of children as between 0 to 11 , and 
have classified patients between 12 and 17 as adolescents $[3,17]$. Among children, separate treatment recommendations have been made for those between $0-4$ and 5-11 years [3]. Three observational studies have compared asthmarelated outcomes between ICS and LTRA agents in the agegroup of 4-11 years, but as a subset of their larger study population $[17,19,20]$. Two of these studies found economic benefits with FP in that FP treatment was associated with a significant reduction in asthma-related costs compared to MON treatment $[17,18]$. The third study did not find any difference between treatments in the risk of an ED visit or hospitalization or use of rescue medications [20].

Existing evidence suggests little comparative effectiveness data between ICS and LTRAs from actual clinical practice settings for children with asthma aged 4-11 years, with inconsistent results noted for sub-analyses done in this pediatric subset. Therefore, the current study was designed to compare asthma-related utilization and economic outcomes for the use of low-dose fluticasone propionate $44 \mathrm{mcg}$ (FP44), an ICS, versus montelukast (MON), an LTRA, in pediatric asthma patients aged 4-11 years.

\section{METHODS}

\section{Data Source}

Administrative claims data from the IMS Life Link Health Plan Claims Database was used for this observational study, which contains data from over 90 different managed healthcare plans encompassing over 60 million lives. Three component files of this database were used: medical (data on diagnostic and therapeutic services rendered in both inpatient and outpatient settings), pharmacy (data on prescription drugs dispensed, with accompanying information on NDC code, quantity, and days' supply), and eligibility (data on demographic characteristics and periods of eligibility for each patient). For both medical and pharmacy files, the dates of service are recorded as well as the amounts charged and paid for the service/drug, whereas the eligibility file contains data on demographic characteristics and periods of eligibility for each patient. Data collection and analysis were in compliance with the Health Insurance Portability and Accountability Act (HIPAA).

\section{Study Design and Patient Selection}

This is a retrospective observational cohort study and designed as a matched cohort analysis (GSK ADA112608). Patients 4-11 years of age with at least 1 pharmacy claim for FP44 or MON during the enrollment period from January 1, 2001 to May 31, 2008 were eligible for study inclusion (Fig. 1). The index date for each patient was defined as the first chronologically occurring pharmacy claim for the study drugs during this period. Patients had to be eligible to receive healthcare services during the 1-year period before the index date (termed the pre-index period) and the 30-day period after the index date (termed the clean period). Patients were also required to have at least one asthma diagnosis (ICD-9 code 493.xx) during the same timeframe. The preindex period was used to assess the baseline characteristics of patients that might be associated with treatment initiation. The clean period was used to ensure receipt of monotherapy for the purpose of categorizing patients into cohorts. Patients were excluded from the analysis if they (1) received any controller medications (including ICSs, LTRAs, cromolyn/ nedocromil, long-acting beta-agonists, combination product of inhaled corticosteroid and long-acting beta-agonists) during the pre-index period or (2) had an asthma-related hospitalization/ED visit or received controller medications other than the study drugs during the clean period.

Patients meeting all selection criteria were then placed into one of two distinct cohorts based on the index prescription: (1) FP44; or (2) MON. Outcomes were then evaluated for patients in both cohorts during the follow-up period defined as the time period between day one after the clean period (day 31) to the first date of any of the following events: 1) therapy change (use of a controller medication other than index medication during the observation period); 2 ) end of continuous eligibility in the health plan; 3 ) end of study period (June 30, 2008); and/or 4) maximum of 365 days of observation.

\section{Propensity Score Matching}

Patients eligible for study inclusion were propensityscore matched in a ratio of one FP44 patient to two MON patients using the technique of nearest available matching on the estimated propensity score, calculated to 3 decimal places $(0.001)$ [21]. The propensity score was obtained from a logistic regression model that predicted the probability of receiving FP44 based on baseline variables including age, gender, United States (U.S.) census region, season at index date (spring, summer, autumn, and winter), physician specialty at index date, Charlson comorbidity index (CCI), presence of concomitant allergic rhinitis, number of shortacting beta-agonist (SABA) canisters, number of oral corticosteroid (OCS) prescriptions, presence of hospital/ED visits for asthma-related conditions, and total asthma-related costs in the pre-index period.

\section{Study Outcomes}

The main clinical outcome was an asthma attack that resulted in an ED visit (primary diagnosis of asthma ICD-9 CM 493.xx). Additionally, a composite measure of an asthma-related hospitalization or ED visit was also assessed. The main economic outcome was asthma-related medical, pharmacy, and total costs calculated on a monthly basis for each patient and adjusted to 2008 \$US based on the medical component of the Consumer Price Index. Asthma-related medical costs were defined by medical claims with a primary diagnosis for asthma (primary discharge diagnosis for asthma-related hospitalization), and asthma-related pharmacy costs included those for both controller and rescue medications. In addition to these two main outcomes, monthly asthma-related rescue medication use, focusing on SABA use and OCS prescriptions was also evaluated.

\section{Analytic Approach}

To examine baseline differences between cohorts, a descriptive analysis of the study sample was conducted using standard summary statistics (eg, means and proportions). For the unmatched population, differences in baseline covariates were compared using t-tests or Mann-Whitney tests for continuous variables and chi-square tests for categorical variables. For the matched population, baseline comparisons were done using paired t-tests or Wilcoxon signed rank tests for continuous and McNemar's test for categorical variables. 
Study Period: Jan 1, 2000 to Jun 30, 2008

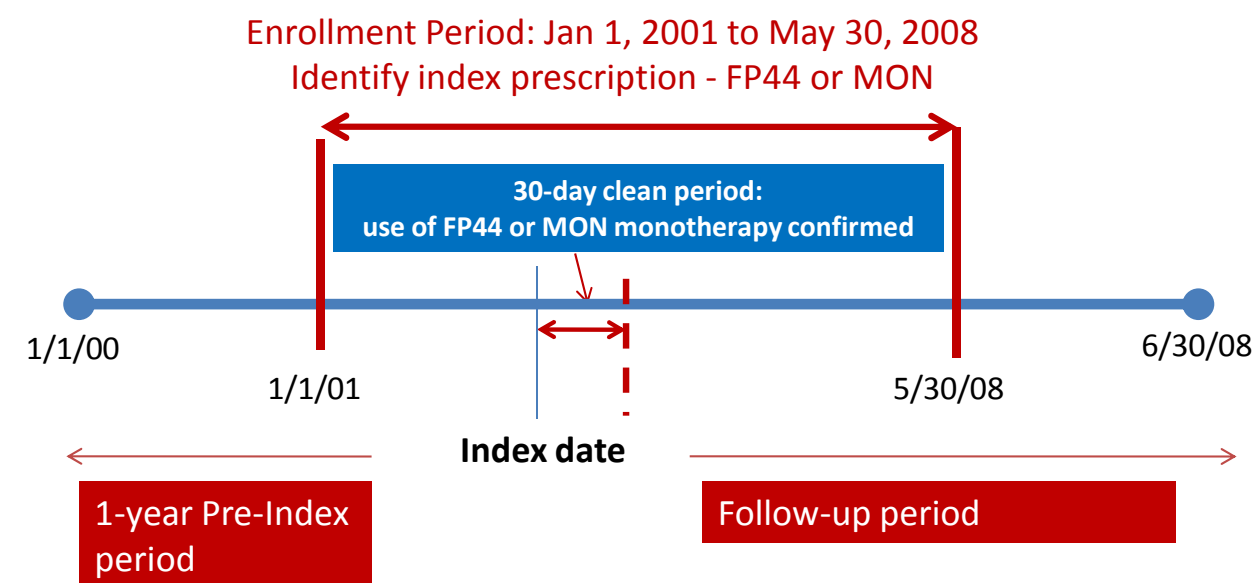

Fig. (1). Study design.

To account for varying time periods, asthma ED rates were calculated by dividing the number of patients who had an asthma attack leading to an ED visit by the total persontime observations (numbers of patients with asthma ED visit $\div$ total person-time). The asthma-related ED visit rate per 100 person-years was then statistically compared using a univariate Cox proportional hazards model. The composite measure of asthma-related hospitalization or ED visit was calculated and statistically compared in a similar fashion. Outcomes of monthly asthma-related medication use and costs were statistically compared using Wilcoxon signed rank tests.

Since the patients were matched on their propensity to receive FP44 based on baseline covariates, it was expected that the analyses of the outcomes would not require any further adjustment, and accordingly the above univariate statistical tests would suffice. However, if an imbalance in any of the baseline covariates was found after matching, then further statistical adjustment was done by incorporating the respective covariate(s) in a multivariate model. The Cox proportional hazards model was used to evaluate differences between cohorts in the risk of an asthma-related ED visit or a composite measure of asthma-related hospitalization/ED visit. Adjusted monthly asthma-related total, pharmacy and medical costs for each cohort were evaluated with 2-part generalized linear models (GLM) with log-link and appropriate distribution, and zero-inflated Poisson models were used to compute adjusted monthly asthma-related rescue medications.

\section{RESULTS}

\section{Study Sample}

A total of 30,986 patients initiated an ICS or a LTRA during the enrollment period and met all study criteria. Of these, 16,884 initiated MON and 2,294 initiated FP44. The cohorts were of similar ages and gender distribution (Table 1). FP44 was more likely to be prescribed during the colder seasons - winter and autumn compared to MON which was more likely to be prescribed during the warmer seasons summer and spring. The cohorts did not differ in terms of overall comorbid burden represented by the CCI. However, a significantly higher proportion of MON-treated patients had a concomitant diagnosis of allergic rhinitis $(46.9 \%$ vs $31.6 \%$; $\mathrm{p}<0.001)$ compared to FP44-treated patients. Both cohorts were similar in all markers of asthma severity except one. The proportion of patients using SABA $(71.3 \%$ vs $43.4 \%$; $\mathrm{p}<0.001)$ and the mean number of canisters $(1.26$ vs 0.75 , $\mathrm{p}<0.001$ ) was found to be significantly higher for the FP44 compared to the MON cohort.

The 1:2 matching process retained 2,212 patients in the FP44 cohort and 4,424 patients in the MON cohort, for which pre-index patient characteristics are summarized in Table 1. Most of the differences between cohorts on variables used for the match were eliminated except that the FP44 cohort still had a significantly higher rate of SABA use in the pre-index period $(81.6 \%$ vs $71.5 \%$; $\mathrm{p}<0.001)$ and mean number of SABA canisters used $(1.29$ vs $1.21 ; \mathrm{p}<0.001)$. These were subsequently used as covariates in all statistical models.

\section{Outcome Measures}

Patients with FP44 had a significantly lower, unadjusted asthma-related ED visit rate per 100 person-years compared to the MON cohort (Table 2: 3.40 vs 4.76 per 100 personyears; $p=0.037$ ). The unadjusted, composite measure of hospitalization/ED visit rate was lower but not significant in the FP compared to the MON cohort. Adjusted survival curves obtained from Cox-proportional hazards model controlling for differences in SABA use at baseline were divergent for both types of events, indicating shorter time to event for the MON compared to the FP44 cohort (Fig. 2). In particular, treatment with FP44 was associated with a $29 \%$ significant reduction in the risk of an ED visit and a $25 \%$ significant reduction in risk of a hospitalization or ED visit compared to treatment with MON (Fig. 2).

A significant $36 \%$ reduction in unadjusted, total asthmarelated costs per month was found related to FP44 treatment initiation (Table 2: $\$ 48$ vs $\$ 75 ; \mathrm{p}<0.001$ ). Differences between cohorts in total costs were driven by differences in asthma-related pharmacy costs per month as similar monthly medical costs were noted between cohorts. Inclusion of baseline SABA use in the multivariate models did not alter 
Table 1. Baseline Cohort Comparison Before and After Propensity Score Matching

\begin{tabular}{|c|c|c|c|c|c|c|}
\hline Characteristics & \multicolumn{3}{|c|}{ Before Match } & \multicolumn{3}{|c|}{ After Match } \\
\hline Female (\%)* & 40.4 & 39.3 & 0.348 & 40.6 & 40.6 & 0.964 \\
\hline \multicolumn{7}{|l|}{ Region (\%)* } \\
\hline Midwest & 57.1 & 45.5 & $<0.001$ & 56.5 & 56.2 & 0.727 \\
\hline South & 4.1 & 14.7 & $<0.001$ & 4.3 & 3.5 & 0.002 \\
\hline West & 5.8 & 9.8 & $<0.001$ & 6.0 & 5.9 & 0.843 \\
\hline \multicolumn{7}{|l|}{ Season of index prescription(\%)* } \\
\hline Summer & 13.1 & 18.1 & $<0.001$ & 13.5 & 12.8 & 0.314 \\
\hline Spring & 26.9 & 30.2 & 0.001 & 27.3 & 26.7 & 0.552 \\
\hline \multicolumn{7}{|l|}{ Physician specialty (\%)* } \\
\hline Specialist & 11.8 & 15.9 & $<0.001$ & 12.3 & 11.7 & 0.433 \\
\hline Primary care & 72.9 & 66.3 & $<0.001$ & 72.5 & 70.8 & 0.078 \\
\hline Others & 15.2 & 17.7 & 0.003 & 15.3 & 17.5 & 0.005 \\
\hline Charlson index in pre-index period (mean, SD)* & $0.98(0.44)$ & $0.98(0.39)$ & 0.718 & $0.98(0.43)$ & $0.98(0.36)$ & 0.804 \\
\hline Concomitant allergic rhinitis $(\%)^{*}$ & 31.6 & 46.9 & $<0.001$ & $32.2 \%$ & 31.2 & 0.162 \\
\hline Hosp/ED for asthma-related conditions (\%)* & 8.0 & 7.6 & 0.521 & 7.8 & 8.4 & 0.607 \\
\hline Hosp/ED for asthma-related conditions (mean, SD) & $0.09(0.36)$ & $0.09(0.34)$ & 0.397 & $0.09(0.36)$ & $0.10(0.37)$ & 0.314 \\
\hline \multicolumn{7}{|l|}{ Asthma-related costs in pre-index period } \\
\hline Medical costs (mean, SD) & $\$ 260(886)$ & $\$ 245(666)$ & 0.420 & $\$ 259(896)$ & $\$ 275(767)$ & 0.349 \\
\hline Pharmacy costs (mean, SD) & $\$ 41(136)$ & $\$ 40(99)$ & 0.912 & $\$ 39(138)$ & $\$ 42(83)$ & 0.127 \\
\hline Total costs (mean, SD) & $\$ 301(905)$ & $\$ 285(453)$ & 0.420 & $\$ 297(914)$ & $\$ 318(785)$ & 0.262 \\
\hline
\end{tabular}

*Variables used for propensity matching. FP44 = Fluticasone propionate 44mcg, MON = montelukast, SD = standard deviation Hosp= hospitalizations, ED = emergency department visit, $\mathrm{AR}=$ allergic rhinitis, $\mathrm{SABA}=$ short acting beta agonists, $\mathrm{OCS}=$ oral corticosteroids.

the results for economic outcomes and consequently, similar results were obtained for adjusted costs (Fig. 3). The cohorts differed in the type of rescue medication use in the follow up period with a significant reduction in OCS use noted for the FP44 compared to the MON cohort (0.03 vs $0.04 ; \mathrm{p}<0.001)$, and similar number of SABA canisters found in both cohorts (Table 2). Similar to adjusted analyses for economic outcomes, no change in results for rescue medication use were noted with significant differences still being retained in the number of OCS prescriptions between cohorts during the follow up period (Difference in OCS prescriptions $=-0.01$; $95 \% \mathrm{CI}$ of difference $=(-0.011,-0.009) ; \mathrm{p}<0.05)$.

\section{DISCUSSION}

In children with asthma aged 4 to 11 years, and naïve to any long-term controller therapy, this study has found initiation with FP44 to be associated with a significant reduction both in asthma-related ED visits and hospitalizations and asthma-related costs per month compared to initiation with MON. These findings reaffirm the recommendations from NAEPP and the Global Initiative for Asthma (GINA) that ICS is the preferred initial controller agent for ages 0 to 4 and 5 to 11 years [18,20]. Additionally, they also align with both randomized controlled trials 
Table 2. Unadjusted Outcomes in the Follow-Up Period by Matched Cohorts

\begin{tabular}{|c|c|c|c|}
\hline Outcomes & FP44 $(N=2,212)$ & $\operatorname{MON}(N=\mathbf{4 , 4 2 4})$ & p-Value \\
\hline \multicolumn{4}{|l|}{ Asthma-Related Events } \\
\hline ED visit rate* & 3.40 & 4.76 & 0.037 \\
\hline Hospitalization/ED visit rate* & 4.07 & 5.29 & 0.075 \\
\hline \multicolumn{4}{|l|}{ Monthly Asthma-Related Costs } \\
\hline Medical costs, mean (SD) & $\$ 26(\$ 165)$ & $\$ 27(\$ 176)$ & 0.852 \\
\hline Pharmacy costs, mean (SD) & $\$ 21(\$ 87)$ & $\$ 48(\$ 75)$ & $<0.001$ \\
\hline Total costs, mean (SD) & $\$ 48(\$ 190)$ & $\$ 75(\$ 197)$ & $<0.001$ \\
\hline \multicolumn{4}{|l|}{ Monthly Rescue Medication Use } \\
\hline Number of SABA canisters, mean (SD) & $0.11(0.24)$ & $0.11(0.26)$ & 0.963 \\
\hline Number of OCS prescriptions, mean (SD) & $0.03(0.10)$ & $0.04(0.04)$ & $<0.001$ \\
\hline
\end{tabular}

*Rate is per 100 person-years. FP 44= Fluticasone propionate $44 \mathrm{mcg}, \mathrm{MON}=$ montelukast, $\mathrm{ED}=$ emergency department, $\mathrm{SD}=$ standard deviation, $\mathrm{SABA}=$ short acting betaagonists, OCS $=$ oral corticosteroids.

\section{A. Time to ED Visit}

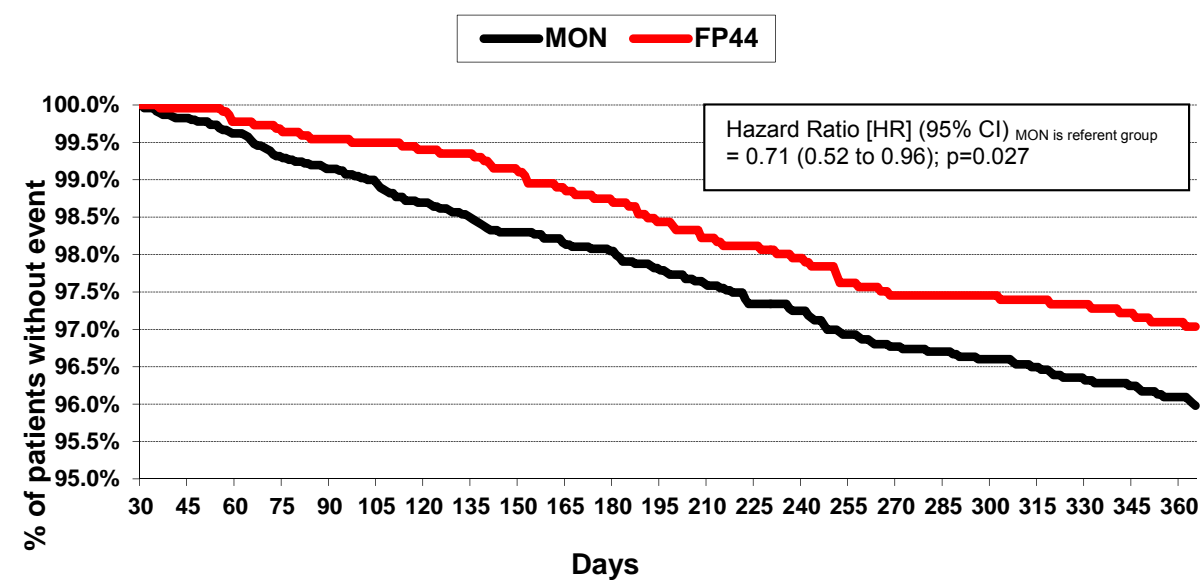

B. Time to Hospitalization/ED Visit

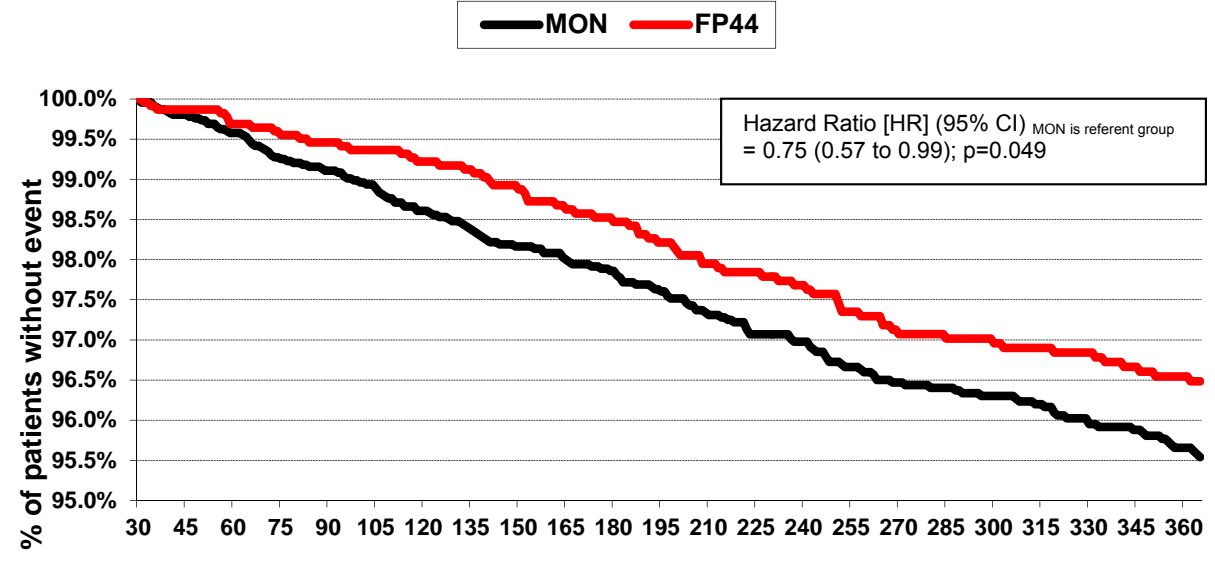

Days

Fig. (2). Adjusted survival curves for (A) time to ED visit and (B) time to hospitalization/ED visit.

specifically evaluating this pediatric age-range and subanalyses of other observational studies [8-14, 17-19].
The current study is the first observational study specifically designed to compare an ICS and LTRA in a 




${ }^{*} \mathrm{p}<0.05$ if difference of the CI estimates does not cross zero.

Fig. (3). Adjusted asthma-related costs per month.

population aged 4 to 11 years. It utilized a large, administrative claims database as the data source allowing for a large patient population to be analyzed. Previous observational studies have included this age subset as part of their larger population that includes adults $(>=18$ years) and/or adolescents (12-17 years), and consequently sample sizes for this age-group were less than 120 in each cohort $[17,18]$. Despite the low sample size in these studies, economic benefits were demonstrated for FP treatment over MON. In one study, the subset of 4-11 years comprised only 86 patients $(\mathrm{FP}=42, \mathrm{MON}=44)$, but still found significantly lower asthma-related charges in the FP compared to the MON cohort in the follow-up period after initiation of these controller medications $(\$ 425$ vs $\$ 932, \mathrm{p}<0.001)$ [18]. Similar results were noted in another study, where patients 4-11 years initiated on low-dose FP (44 mcg) had 30\% lower asthma-related costs of those initiated on MON [17]. Our large study is validation of the findings from these small studies as our cost reduction estimate is within the range of those found in these studies $(36 \%)$.

Our study results differ from the results of another study that evaluated the risk of an ED visit or hospitalization between fluticasone propionate and montelukast in a subanalysis in children aged 6-14 years [20]. This study concluded that both treatments were similar as outcomes were not statistically significant. However, it is noteworthy that the fluticasone propionate group in that study had a lower proportion of patients with an asthma-related ED visit and hospitalization compared to the montelukast group (ED: $4.8 \%$ vs $6.2 \%, \mathrm{p}=0.22$, and 0.6 s $1.6, \mathrm{p}=0.09$ ). Furthermore, this age sub-group analysis was not sufficiently powered to find a difference as acknowledged by the authors [20]. Our finding of a significant reduction in the risk of asthmarelated hospitalization/ED visits is particularly notable considering our study design. We used the variable followup approach which is a particular strength of the study as it allows outcomes to be attributed to the treatment and not be influenced by downstream changes in treatment such as a switch or augmentation of controller therapy among other conditions (see Methods). Thus, the event of ED visits or hospitalization was ascertained before the censor date. If patients with MON were more likely to have switched or augmented their controller therapy as has been seen in other studies, then they would have a shorter follow-up period during which events could be analyzed as per our design [19]. Despite this, a higher rate of ED visits or hospitalizations was seen in the MON compared to the FP44 cohort in our study.

The current study is not without limitations, and a discussion of these is warranted to assist with results' interpretation. Randomized controlled trials share an inherent advantage in that the process of randomization accounts for unobservable confounders that balances the cohorts at baseline before subsequent comparisons can be made. The current study matched cohorts on propensity scores to mimic this balance between cohorts despite similarities in several markers of asthma severity at baseline before matching (see Table 1) [21]. This is a notable strength of the current study. However, this approach is limited by the ability to only include measures that are available in the dataset. Thus, variables that are known to be associated with outcomes but could not be measured in the dataset (eg pulmonary function measures, socioeconomic status, race) might confound study results. Although pulmonary function measures were not available in the data for comprehensive assessment of asthma severity, surrogate measures were used including SABA use, OCS use, asthma-related hospitalization/ED visit rate, and asthma-related costs. If these measures were unable to adequately capture asthma severity, it is possible that residual confounding may exist. Lastly, we assumed that a prescription filled was taken. This affects our assessment of rescue medication use if patients only filled but did not end up taking the medication.

The current study represents an important addition to the evidence-base of comparative effectiveness research in the treatment of pediatric asthma. The study population and dosages of the study treatment used are relevant to the practicing clinician to evaluate the significance of the study results. In particular, children who are naïve to controller therapy will benefit from initiating therapy first with ICS (FP) than with LTRA. Further, the low-dose of ICS evaluated in this study is relevant to the pediatric population 
and is in accordance with NAEPP recommendations [18]. In conclusion, significant reductions in asthma-related ED visits and hospitalization, asthma-related total costs, and OCS use can be realized if long-term controller therapy is initiated with FP44 compared to MON in children aged 4 to 11 years.

\section{ACKNOWLEDGEMENT}

Funding for this study and resultant publication was provided by GlaxoSmithKline (GSK).

\section{CONFLICT OF INTEREST}

Dr. Stanford is an employee of GSK.

\section{REFERENCES}

[1] Akinbami L. National Center for Health Statistics - Health E-Stat. Asthma prevalence, health care use and mortality: United States 2003-2005. Available at: http://www.cdc.gov/nchs/data/hestat/ asthma03-05/asthma03-05.htm\#ed_visits [Accessed October 10, 2011].

[2] Care of Children and Adolescents in U.S. Hospitals. HCUP Fact Book No. 4. AHRQ Publication No. 04-0004, October 2003. Agency for Healthcare Research and Quality, Rockville, MD. Available at: http://www.ahrq.gov/data/hcup/factbk4/factbk4.htm [Accessed October 10, 2011].

[3] National Asthma Education and Prevention Program. Expert Panel Report 3 (EPR-3): Guidelines for the Diagnosis and Management of Asthma-Summary Report 2007. J Allergy Clin Immunol 2007; 120(5 Suppl): S94-138.

[4] From the Global Strategy for Asthma Management and Prevention, Global Initiative for Asthma (GINA) 2010. Available at: http://www.ginasthma.org/ [Accessed October 10, 2011].

[5] Peden DB, Berger WE, Noonan MJ, et al. Inhaled fluticasone propionate delivered by means of two different multidose powder inhalers is effective and safe in a large pediatric population with persistent asthma. J Allergy Clin Immunol 1998; 102(1): 32-8.

[6] Katz Y, Lebas FX, Medley HV, Robson R. Fluticasone propionate 50 micrograms BID versus 100 micrograms BID in the treatment of children with persistent asthma. Fluticasone Propionate Study Group. Clin Ther 1998; 20(3): 424-37.

[7] Adams RJ, Fuhlbrigge A, Finkelstein JA, et al. Impact of inhaled antiinflammatory therapy on hospitalization and emergency department visits for children with asthma. Pediatrics 2001; 107(4): 706-11.
[8] Garcia Garcia ML, Wahn U, Gilles L, Swern A, Tozzi CA, Polos P Montelukast, compared with fluticasone, for control of asthma among 6- to 14-year-old patients with mild asthma: the MOSAIC study. Pediatrics 2005; 116(2): 360-9.

[9] Jartti T. Inhaled corticosteroids or montelukast as the preferred primary long-term treatment for pediatric asthma? Eur J Pediatr 2008; 167(7): 731-6.

[10] Kooi EM, Schokker S, Marike Boezen H, et al. Fluticasone or montelukast for preschool children with asthma-like symptoms: Randomized controlled trial. Pulm Pharmacol Ther 2008; 21(5): 798-804.

[11] Ostrom NK, Decotiis BA, Lincourt WR, et al. Comparative efficacy and safety of low-dose fluticasone propionate and montelukast in children with persistent asthma. J Pediatr 2005; 147(2): 213-20.

[12] Szefler SJ, Phillips BR, Martinez FD, et al. Characterization of within-subject responses to fluticasone and montelukast in childhood asthma. J Allergy Clin Immunol 2005; 115(2): 233-42.

[13] Sorkness CA, Lemanske RF Jr, Mauger DT, et al. Long-term comparison of 3 controller regimens for mild-moderate persistent childhood asthma: the pediatric asthma controller trial. J Allergy Clin Immunol 2007; 119(1): 64-72.

[14] Zeiger RS, Szefler SJ, Phillips BR, et al. Response profiles to fluticasone and montelukast in mild-to-moderate persistent childhood asthma. J Allergy Clin Immunol 2006; 117(1): 45-52.

[15] Ducharne FM, DiSalvio F. Anti-leukotriene agents compared to inhaled corticosteroids in the management of recurrent and/or chronic asthma in adults and children. Cochrane Database Syst Rev 2005; CD005307.

[16] Concato J, Shah N, Horwitz RI. Randomized, controlled trials, observational studies, and the hierarchy of research designs. N Engl J Med 2000; 324; 1887-92.

[17] Stempel DA, Mauskopf J, McLaughlin T, Yazdani C, Stanford RH. Comparison of asthma costs in patients starting fluticasone propionate compared to patients starting montelukast. Respir Med 2001; 95(3): 227-34.

[18] Pathak DS, Davis EA, Stanford RH. Economic impact of asthma therapy with fluticasone propionate, montelukast, or zafirlukast in a managed care population. Pharmacotherapy 2002; 22(2): 166-74.

[19] Stempel DA, Kruzikas DT, Manjunath R. Comparative efficacy and cost of asthma care in children with asthma treated with fluticasone propionate and montelukast. J Pediatr 2007; 150(2): 162-7.

[20] Allen-Ramey FC, Markson LE, Riedel AA, Sajjan S, Weiss KB. Patterns of asthma-related health care resource use in children treated with montelukast or fluticasone. Curr Med Res Opin 2006; 22(8): 1453-61.

[21] Rubin DB. Estimating causal effects from large data sets using propensity scores. Ann Intern Med 1997; 127: 757-63. 